\title{
Quasinormal mode analysis in BEC acoustic black holes
}

\author{
C. Barceló, ${ }^{1}$ A. Cano, ${ }^{1}$ L. J. Garay, ${ }^{2,3}$ and G. Jannes ${ }^{1,3}$ \\ ${ }^{1}$ Instituto de Astrofísica de Andalucía, CSIC, Camino Bajo de Huétor 50, 18008 Granada, Spain \\ ${ }^{2}$ Departamento de Física Teórica II, Universidad Complutense de Madrid, 28040 Madrid, Spain \\ ${ }^{3}$ Instituto de Estructura de la Materia, CSIC, Serrano 121, 28006 Madrid, Spain
}

(Received 5 February 2007; published 13 April 2007)

\begin{abstract}
We perform a quasinormal mode analysis of black-hole configurations in Bose-Einstein condensates (BECs). In this analysis we use the full Bogoliubov dispersion relation, not just the hydrodynamic or geometric approximation. We restrict our attention to one-dimensional flows in BECs with steplike discontinuities. For this case we show that in the hydrodynamic approximation quasinormal modes do not exist. The full dispersion relation, however, allows the existence of quasinormal modes. Remarkably, the spectrum of these modes is not discrete but continuous.
\end{abstract}

DOI: $10.1103 /$ PhysRevD.75.084024

PACS numbers: 04.70.Dy, 03.75.Kk, 04.80.-y

\section{INTRODUCTION}

Analogue models of gravity $[1,2]$ allow the study of certain geometrical aspects of linearized general relativity (GR). When the analogue models are based on an intrinsically quantum system, such as Bose-Einstein condensates (BECs) [3-5], this opens up the possibility of studying some corrections to this geometric description. These corrections - encoded in the form of nonrelativistic or modified dispersion relations, similar to what is expected in a wide range of scenarios for gravity at Planck-scale energies, leading to possible violations of Lorentz invariance [6]- are then of a quantum origin, but they express themselves in a classical manner. In general, these corrections have to be taken into account in any treatment involving high energies (high frequencies). In BECs, in particular, in the presence of horizons, the deviations from the geometric or hydrodynamic regime are important even at low energies [7].

In a previous article [7], the stability of several onedimensional configurations in BECs was studied. These configurations were chosen in analogy with the geometries associated with gravitational black holes. Here we will elaborate further on black-hole configurations, which were shown to be devoid of instabilities, and examine their quasinormal or relaxation modes. The analysis we carry out is analogous to the standard quasinormal mode (QNM) analysis in gravity [8,9]. According to GR, when black holes are perturbed, they emit gravitational waves in the following way. The initial regime depends mainly on the concrete form of the perturbation. Then, an oscillatory phase is attained in which the precise form of the emitted wave depends only on the properties of the black hole itself. Finally, a polynomial tail characterizes the return to equilibrium. In the intermediate phase, a discrete set of complex frequencies are excited: the quasinormal modes of our concern. Since quasinormal modes are modes of decay, i.e. of energy dissipation, they are outgoing. In GR this means that their group velocities are directed outwards both in the asymptotic region and at the horizon. Moreover, as we will illustrate, in GR this also automatically implies that they are non-normalizable or divergent.

A general investigation of quasinormal modes in analogue black holes was carried out in Ref. [10]. As we will briefly point out, in standard GR in $(1+1)$ dimensions, quasinormal modes do not exist. In higher dimensions, a discrete spectrum appears [8,9]. Similar results were found in Ref. [10] for analogue black holes. Here the essential element that we add is the influence of a nonrelativistic contribution to the dispersion relation on these QNMs. ${ }^{1}$ With the full Bogoliubov dispersion relation in a BEC, not only does a QNM-spectrum appear even in the onedimensional case studied here, but this spectrum turns out to be continuous. This result suggests the straightforward speculation that in $(3+1)$ dimensions, for both analogue and GR black holes, there will also be continuous regions of QNMs when modifications of the dispersion relation with respect to the hydrodynamic or relativistic case are taken into account.

The structure of this paper is as follows. In the next two sections we will review the steps that lead from the general description of a BEC to the simplified formalism for onedimensional configurations with abrupt discontinuities, and highlight the modified dispersion relation. In Sec. IV we will discuss the boundary conditions for our problem, and compare with the standard quasinormal mode analysis of gravitational black holes. Then, in Sec. V we present and discuss our main results.

\footnotetext{
${ }^{1}$ Modifications of the quasinormal mode spectrum of black holes due to Lorentz violations have also been investigated in Ref. [11] but from a different perspective. They introduce spontaneous Lorentz-symmetry breaking terms in an effective Lagrangian via nonzero expectation values of different tensor fields in the vacuum, thereby restricting attention to a modified Dirac equation in a Schwarzschild background. However, these terms do not lead to modified dispersion relations of the type analyzed here.
} 


\section{PRELIMINARIES}

In this section we will briefly review the description of background BEC configurations and their perturbations, with particular emphasis on the modified dispersion relation. The main aim here is to fix notation. General reviews of BECs can be found e.g. in Refs. $[12,13]$ while the type of configurations discussed here were amply described in Ref. [7].

A dilute gas of interacting bosons can be described in terms of quantum field operators $\hat{\Psi}(\mathbf{x})$ [and $\left.\hat{\Psi}^{\dagger}(\mathbf{x})\right]$ that annihilate [and create] particles. When this gas condensates, $\hat{\Psi}$ can be separated into a macroscopic wave function $\psi$ and a field operator $\hat{\varphi}$ describing quantum fluctuations: $\hat{\Psi}=\psi+\hat{\varphi}$. The macroscopic wave function $\psi$ satisfies the Gross-Pitaevskii (GP) equation

$$
i \hbar \frac{\partial}{\partial t} \psi(t, \mathbf{x})=\left(-\frac{\hbar^{2}}{2 m} \nabla^{2}+V_{\mathrm{ext}}(\mathbf{x})+g|\psi|^{2}\right) \psi(t, \mathbf{x})
$$

where $m$ is the boson mass, $V_{\text {ext }}$ the external potential and $g$ a coupling constant which is related to the corresponding scattering length $a$ through $g=4 \pi \hbar^{2} a / m$.

This equation can be expressed in terms of hydrodynamic quantities such as the local speed of sound $c$ and the velocity of the fluid flow $\mathbf{v}$. This proceeds by first introducing the Madelung representation for the order parameter in the GP equation:

$$
\psi=\sqrt{n} e^{i \theta / \hbar} e^{-i \mu t / \hbar} .
$$

Here $n$ is the condensate density, $\mu$ the chemical potential and $\theta$ a phase factor. Next, as we are interested in the acoustic perturbations of the BEC, we linearize in $n$ and $\theta$ :

$$
\begin{aligned}
& n(\mathbf{x}, t)=n_{0}(\mathbf{x})+g^{-1} \tilde{n}_{1}(\mathbf{x}, t), \\
& \theta(\mathbf{x}, t)=\theta_{0}(\mathbf{x})+\theta_{1}(\mathbf{x}, t) .
\end{aligned}
$$

Then, the following set of equations for the linear perturbations is obtained from the complex GP equation:

$$
\begin{aligned}
& \partial_{t} \tilde{n}_{1}=-\nabla \cdot\left(\tilde{n}_{1} \mathbf{v}+c^{2} \nabla \theta_{1}\right), \\
& \partial_{t} \theta_{1}=-\mathbf{v} \cdot \nabla \theta_{1}-\tilde{n}_{1}+\frac{1}{4} \xi^{2} \nabla \cdot\left[c^{2} \nabla\left(\frac{\tilde{n}_{1}}{c^{2}}\right)\right],
\end{aligned}
$$

which are equivalent to the Bogoliubov equations, and where $\xi \equiv \frac{\hbar}{m c}$ is the healing length, and $c$ and $\mathbf{v}$ are

$$
c^{2} \equiv g n_{0} / m, \quad \mathbf{v} \equiv \nabla \theta_{0} / m
$$

The last term on the right-hand side in $(2.4 \mathrm{~b})$ is the socalled "quantum potential" term.

At this point, let us briefly recall that in the hydrodynamic limit, which is obtained by neglecting the quantum potential term just mentioned, an effective Lorentzian metric can be constructed of the type

$$
\left(\mathrm{g}_{\mu \nu}\right) \propto\left(\begin{array}{cc}
v^{2}-c^{2} & -\mathbf{v}^{\mathrm{T}} \\
-\mathbf{v} & \mathbb{1}
\end{array}\right),
$$

which historically formed the onset of the interest in hydrodynamic analogue models for gravity (for an overview, see Ref. [1] and references therein).

From now on, consider one-dimensional profiles. The Eqs. (2.4) can be solved in a homogeneous region ( $c$ and $v$ constant) by writing the perturbations in plane-wave form

$$
\begin{aligned}
& \tilde{n}_{1}(x, t)=A e^{i(k x-\omega t)}, \\
& \theta_{1}(x, t)=B e^{i(k x-\omega t)} .
\end{aligned}
$$

This immediately leads to the local dispersion relation ${ }^{2}$

$$
(\omega-v k)^{2}=c^{2} k^{2}+\frac{1}{4} c^{2} \xi^{2} k^{4} .
$$

This dispersion relation is quartic in $k$. It should be compared to the hydrodynamic dispersion relation that is obtained by neglecting the quantum potential:

$$
(\omega-v k)^{2}=c^{2} k^{2} .
$$

This hydrodynamic dispersion relation is of the usual quadratic form.

However, here we are not so much interested in this hydrodynamic limit, but precisely in deviations from it. Therefore we will always work with the full dispersion relation, and only use the hydrodynamic limit as a means of comparison. The key point is that, because the full dispersion relation is quartic, when writing the mode corresponding to a certain frequency $\omega$

$$
u_{\omega}=e^{-i \omega t} \sum_{j} A_{j} e^{i k_{j} x},
$$

there are now four contributions to this $u_{\omega}$-mode, stemming from the four values of $k$ associated to each value of $\omega$. In the hydrodynamic limit there are obviously only two contributions to each $u_{\omega}$-mode:

$$
k_{1,2}=\omega /(v \pm c) .
$$

In the configurations analyzed in this paper, the additional, "nonhydrodynamic" modes will turn out to play a crucial role even at low frequencies.

\section{BACKGROUND CONFIGURATIONS AND NUMERICAL METHOD}

We are looking for quasinormal modes of black-hole configurations in one-dimensional BECs of the type represented in Fig. 1. These profiles are piecewise homogeneous with a single discontinuity. In order to find these

\footnotetext{
${ }^{2}$ The term "dispersion relation" is sometimes reserved strictly for an equation of the form $\omega=\omega(k)$, while an equation such as Eq. (2.8) would then have to be called, e.g., the "characteristic equation." For the sake of clarity, we will always use the term dispersion relation to refer to an equation of the form (2.8) or (2.9).
} 

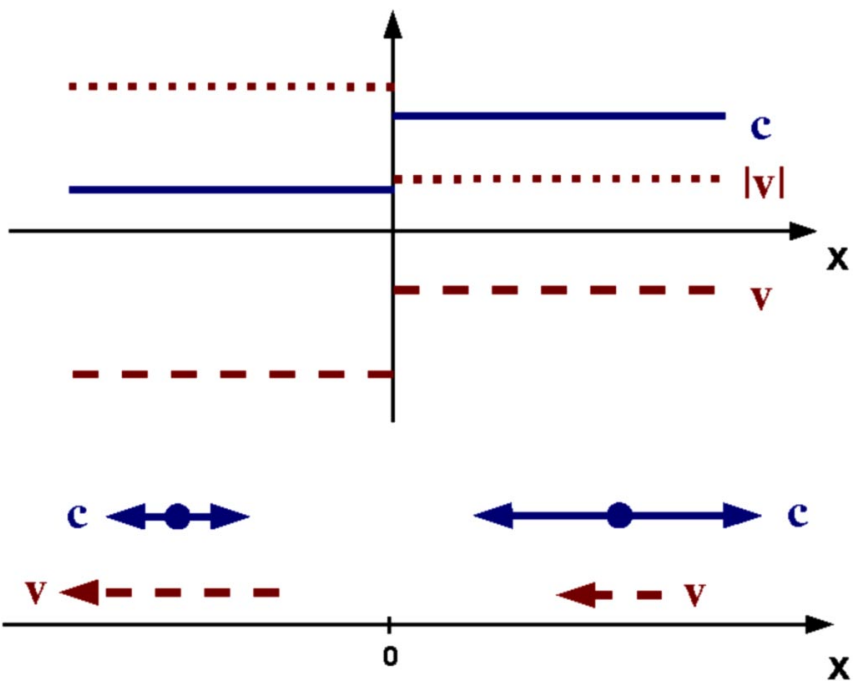

FIG. 1 (color online). Flow and sound velocity profile consisting of two homogeneous regions with a steplike discontinuity at $x=0$ simulating a black-holelike configuration in a BEC. The solid blue line represents the speed of sound $c$, the dashed red line the fluid velocity $v$. In the upper part of the picture, the negative value of $v$ indicates that the fluid is left-moving. For $x>0$, the fluid is subsonic since $c>|v|$. At $x<0$ it has become supersonic. At $x=0$, there is a sonic horizon.

relaxation modes, first, we will need a way to describe the mode solutions that characterize the profiles under study. The idealized form of these profiles allows a simplified description in each homogeneous region. Then, the various sections have to be linked through matching conditions at the discontinuity. After finding all the mode solutions, the quasinormal modes will be those that moreover satisfy the adequate boundary conditions. We will describe the matching conditions in the current section, together with a straightforward algorithm for numerical implementation. The boundary conditions will be the topic of the next section.

Integrating (2.4) in an infinitesimal neighborhood of the discontinuity (say $x=0$ ) and simplifying with the use of the continuity equation ( $v c^{2}=$ const) leads, for our choice of a piecewise homogeneous background, to the following set of matching conditions [7]:

$$
\begin{array}{ll}
{\left[\theta_{1}\right]=0,} & {\left[c^{2} \partial_{x} \theta_{1}\right]=0,} \\
{\left[\frac{\tilde{n}_{1}}{c^{2}}\right]=0,} & {\left[\partial_{x} \tilde{n}_{1}\right]=0,}
\end{array}
$$

where the brackets indicate, for example, $\left[\theta_{1}\right]=$ $\left.\theta_{1}\right|_{x=0^{+}}-\left.\theta_{1}\right|_{x=0^{-}}$.

In each homogeneous section, we look for a solution for a particular frequency $\omega$ as a sum of $k$-modes of the form

$$
\tilde{n}_{1}=\sum_{j} A_{j} e^{i\left(k_{j} x-\omega t\right)}
$$

$$
\theta_{1}=\sum_{j} A_{j} \frac{\omega-v k_{j}}{i c^{2} k_{j}^{2}} e^{i\left(k_{j} x-\omega t\right)},
$$

where the sum is over the four $k_{j}$-values associated to $\omega$ in that homogeneous region.

The conditions (3.1) can be written in matrix form

$$
\Lambda_{i j} A_{j}=0 .
$$

Since there are eight free parameters $A_{j}$ for each discontinuity (four in each region), and only four conditions, matching is in principle always possible. However, there are additional conditions: the boundary conditions which we will discuss in the next section. These boundary conditions can be added to the matrix $\Lambda_{i j}$, to obtain a (4+ $N) \times 8$ matrix $\tilde{\Lambda}_{i j}$, with $N$ the number of constraints on the coefficients $A_{j}$ that follow from the boundary conditions. Depending on the value of $N$, we can have the following cases.

(i) If $N<4$, then there will always exist a nontrivial solution $A_{j}$, since there are more degrees of freedom than constraints. Therefore, a region of the complex $\omega$-plane where $N<4$ will represent a continuous region of eigenfrequencies.

(ii) If $N=4$, then we have a $8 \times 8$ system of equations and there is a nontrivial solution $A_{j}$ only if $\operatorname{det}(\tilde{\Lambda})=$ 0 . Generally speaking, this means that in a region where $N=4$, we might at the very most expect isolated discrete eigenfrequencies, but no continuous zones of solutions.

(iii) If $N>4$ then the system can be split into two (or more) $8 \times 8$ subsystems, each with a subdeterminant $\lambda_{i}$. For a nontrivial solution to exist, each of these subsystems should fulfill the previous condition $\operatorname{det}\left(\lambda_{i}\right)=0$.

To summarize, we can define a non-negative function $F(\omega)$ such that

(i) $F(\omega)=0$ if $N<4$

(ii) $F(\omega)=|\operatorname{det}(\tilde{\Lambda})|$ if $N=4$,

(iii) $F(\omega)=\sum\left|\operatorname{det}\left(\lambda_{i}\right)\right|$ if $N>4$.

The eigenfrequencies of the system are those values of $\omega$ for which $F(\omega)=0$, and since the quasinormal modes are relaxation modes they will be located in the lower half complex $\omega$ plane.

\section{QUASI-NORMAL MODE ANALYSIS AND BOUNDARY CONDITIONS}

The essential ingredient that is still missing to calculate the quasinormal modes in these BEC black-hole configurations are the boundary conditions. We will first take a look at the boundary conditions that are used to calculate QNMs in GR. This can be immediately extrapolated to BECs in the hydrodynamic limit. However, as we will 
show, in this hydrodynamic limit in $(1+1)$ dimensions, there exist no QNMs. Finally, we will come to the boundary conditions with the full dispersion relation.

\section{A. Quasinormal modes of a gravitational black hole}

Quasinormal modes are simply the modes of energy dissipation of a perturbed object. Gravitational black-hole QNMs, in particular, are the relaxation modes that characterize the pulsations of the black hole after perturbations initiated internally in the spacetime surrounding the black hole.

The fact that these modes decay in time means that they can be represented by complex frequencies $\omega$ with $\operatorname{Im}(\omega)<0$. The fact that they are relaxation modes means that they will have to be outgoing in the asymptotic region. At the horizon, QNMs are also required to be outgoing because in GR nothing can escape through the horizon (note that in QNM terminology "outgoing" means directed towards the exterior of the spacetime region connected to an asymptotic observer, in other words at the horizon this means directed towards the singularity). So the boundary conditions that are imposed in GR to find the QNMs of a black hole are simply that these modes should be outgoing both in the asymptotic region and at the horizon.

Since these QNMs are outgoing, they are also divergent. Indeed, because of the quadratic dispersion relation, say $\omega^{2}=c^{2} k^{2}$, we have for the group velocity $v_{g}$ and for the imaginary part of $k$ :

$$
v_{g}= \pm c, \quad \operatorname{Im}(k)=\operatorname{Im}(\omega) / v_{g}
$$

and, therefore, since QNMs have $\operatorname{Im}(\omega)<0$ :

$$
\operatorname{sign}[\operatorname{Im}(k)]=-\operatorname{sign}\left[v_{g}\right] .
$$

To sum up, QNMs in a gravitational black hole are found by imposing the boundary condition that they should be outgoing, and because of the quadratic dispersion relation they are also automatically divergent.

\section{B. BEC hydrodynamic limit: Absence of quasinormal modes in one-dimensional flows}

In BECs in the hydrodynamic limit, decaying outgoing modes are also automatically divergent, again because of the quadratic dispersion relation. Indeed,

$$
v_{g}=v \pm c, \quad \operatorname{Im}(k)=\operatorname{Im}(\omega) / v_{g}
$$

and therefore $\operatorname{sign}[\operatorname{Im}(k)]=-\operatorname{sign}\left[\boldsymbol{v}_{g}\right]$ as before. Let us see whether QNMs can exist in this case.

In the hydrodynamic limit, the quantum potential term in Eq. (2.4) is neglected, and so upon integration of the remaining terms in these equations, only two matching conditions remain:

$$
\left[\theta_{1}\right]=0, \quad\left[v \tilde{n}_{1}+c^{2} \partial_{x} \theta_{1}\right]=0 .
$$

Note that this second equation cannot be simplified as in Eq. (3.1), because this requires the use of a third matching condition which came precisely from integration of the quantum potential.

The hydrodynamic solution for a particular frequency $\omega$

$$
\begin{aligned}
& \tilde{n}_{1}=A_{1} e^{i\left(k_{1} x-\omega t\right)}+A_{2} e^{i\left(k_{2} x-\omega t\right)}, \\
& \theta_{1}=B_{1} e^{i\left(k_{1} x-\omega t\right)}+B_{2} e^{i\left(k_{2} x-\omega t\right)},
\end{aligned}
$$

has to satisfy $A_{j}=i\left(\omega-v k_{j}\right) B_{j}$. Assume that the configuration is completely subsonic. Then, for $x<0, k_{1}=$ $\omega /(v+c) \equiv k_{\text {in }}^{-}$is ingoing, whereas $k_{2}=\omega /(v-c) \equiv$ $k_{\text {out }}^{-}$is outgoing, and vice versa for $x>0$, where we write $k_{\text {in }}^{+}$and $k_{\text {out }}^{+}$respectively. In terms of the associated coefficients $B_{j}$, the matching conditions then become

$$
\begin{gathered}
B_{\text {out }}^{+}+B_{\text {in }}^{+}=B_{\text {in }}^{-}+B_{\text {out }}^{-}, \\
c^{+}\left(B_{\text {out }}^{+}-B_{\text {in }}^{+}\right)=c^{-}\left(B_{\text {in }}^{-}-B_{\text {out }}^{-}\right),
\end{gathered}
$$

or in matrix form

$$
\left(\begin{array}{c}
B_{\text {in }}^{+} \\
B_{\text {out }}^{+}
\end{array}\right)=\frac{1}{2 c^{+}}\left(\begin{array}{cc}
c^{+}-c^{-} & c^{+}+c^{-} \\
c^{+}+c^{-} & c^{+}-c^{-}
\end{array}\right)\left(\begin{array}{c}
B_{\text {in }}^{-} \\
B_{\text {out }}^{-}
\end{array}\right) .
$$

For a solution to be outgoing in both regions, both $B_{\text {in }}^{+}$and $B_{\text {in }}^{-}$should vanish. This means that the coefficient $c^{+}+c^{-}$ connecting $B_{\text {in }}^{+}$with $B_{\text {out }}^{-}$must be zero. But since $c$ is always a positive integer, this is impossible. Therefore, there are no quasinormal solutions in the hydrodynamic limit.

The above calculation is strictly valid only for completely subsonic profiles. When the left-hand side is supersonic, the hydrodynamic approximation breaks down in the passage through the sonic horizon [7]. Both modes in the supersonic region become outgoing at $x \rightarrow-\infty$, while there is still one outgoing mode in the right asymptotic (subsonic) region. Since the matrix in Eq. (4.6) is well defined, it seems then that some QNMs exist for these configurations. However, this is a spurious effect caused by the subsonic to supersonic steplike discontinuity in combination with the hydrodynamic regime. Precisely because of the presence of the horizon, we know that none of the modes at the left-hand side can be connected to the outgoing mode at the right-hand side. So QNMs cannot exist. From a point of view more similar to GR, when the $(x<0)$ region is supersonic, it becomes disconnected from the subsonic region $(x>0)$. But then, just like in GR, the requirement that QNMs must be outgoing in the left asymptotic region should be replaced by the requirement of being outgoing at the horizon. Because of the homogeneity of the profile, none of the modes can be outgoing both at the right asymptotic region and at the horizon. So in any case, QNMs cannot exist in the hydrodynamic limit in $(1+$ 1) dimensions, regardless of the sub- or supersonic character of the profile. 
Note that in GR in $(1+1)$ dimensions, a more general argument prohibits the existence of QNMs. Indeed, all two-dimensional metrics are conformally flat. In addition, the d'Alembertian equation in two dimensions is conformally invariant [14]. Therefore, all the solutions of the system are conformally equivalent to plane waves, and so they do not satisfy the requirement of being outgoing both in the asymptotic region and at the horizon. Hence QNMs cannot exist in $(1+1)$ GR. The situation is completely different in $(3+1)$ dimensions, in which GR black holes exhibit a discrete QNM spectrum.

\section{Boundary conditions with the full dispersion relation}

As we just discussed, in BECs in the hydrodynamic limit, decaying outgoing modes are automatically divergent. But when looking at the full dispersion law, this relation is no longer valid. Note that for our choice of profiles, we have two asymptotic regions, and moreover no prohibition of crossing the horizon in any direction, since the permeability of the horizon is an essential feature of modified dispersion relations [7]. An outgoing mode is then one that has a group velocity $v_{g}<0$ for $x<0$ and $v_{g}>0$ for $x>0$, where $v_{g}$ is obtained from the full dispersion relation:

$$
v_{g}(k) \equiv \operatorname{Re}\left(\frac{d \omega}{d k}\right)=\operatorname{Re}\left(\frac{c^{2} k+\frac{1}{2} \xi^{2} c^{2} k^{3}}{\omega-v k}+v\right) .
$$

Clearly, there is no immediate connection with the sign of $\operatorname{Im}(k)$ anymore, so the automatic divergent character of outgoing modes is lost.

In any case, quasinormal modes should be outgoing, and this remains the essential boundary condition. Additionally, one might wonder whether the QNMs will be convergent, or divergent after all (in spite of the modification of the dispersion relation). An easy way to check this is by applying convergence as an additional boundary condition and comparing the resulting spectrum with the one obtained without this additional condition.

To sum up, quasinormal modes should be outgoing. In GR and in the hydrodynamic approximation, this automatically implies a divergent behavior in the asymptotic regions. However, in both these cases, there are no QNMs in $(1+1)$ dimensions [as opposed to $(3+1)$ GR black holes with their discrete QNM spectrum]. In BECs with the full modified dispersion relation, outgoing modes are no longer automatically divergent. The boundary condition to determine QNMs in the $(1+1)$ BEC black-hole configurations under study is therefore that they should be outgoing. Nonetheless, we will check whether they are convergent or divergent.

\section{RESULTS AND DISCUSSION}

In this section we present and discuss results for the quasinormal mode spectrum of black holes in one- dimensional BECs obtained with the full dispersion relations.

Figure 2 illustrates the QNM spectrum for an acoustic black hole containing a single discontinuity, subsonic at the right-hand side and supersonic at the left. Remember from Ref. [7] that such a black hole did not possess unstable eigenfrequencies, so it makes sense to look for stable modes.

As discussed in the previous section, the boundary condition that determines the existence of QNMs is that they should be outgoing in both asymptotic regions. Surprisingly, as can be seen from Fig. 2, not only does such a QNM spectrum indeed show up, but this spectrum is actually continuous.

The next question is whether these modes are convergent or not. In Fig. 3, we have imposed convergence, respectively, at both sides, in the right asymptotic region only, and in the left asymptotic region (the singularity) only. The QNM spectrum disappears as soon as the convergence condition is imposed at either side. In other words, the quasinormal modes are divergent. Remember from the previous section that for outgoing hydrodynamic modes, this divergent character is automatic. However, we have also seen that when only retaining the hydrodynamic modes, there are no QNMs. So although the QNMs that we have just found are divergent, the inclusion of the nonhydrodynamic modes is essential for their existence, and so their divergent character is not "automatic," i.e. it is not due to a strict relation between the signs of $v_{g}$ and $\operatorname{Im}(k)$. As a matter of fact, we have checked that the QNMs disappear when the convergent $k$ contributions are excluded.

We have also calculated the QNM spectrum for other black-holelike configurations that were shown to be devoid of instabilities in Ref. [7], and we have checked that a similar continuous spectrum of QNMs appeared in all of
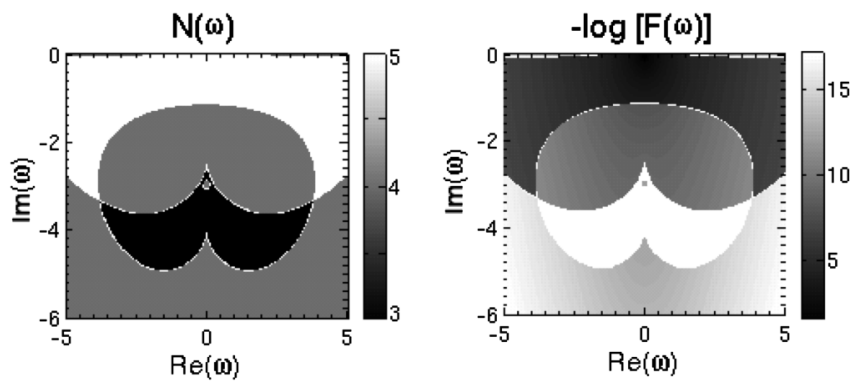

FIG. 2. Plots illustrating the quasinormal mode spectrum for a one-dimensional black-hole configuration in BEC. The left-hand part of the picture represents the number $N$ of constraints following from the boundary condition that QNMs should be outgoing. The right-hand part shows the function $\log [F(\omega)]$ defined in Sec. III, where $F(\omega)=0$ corresponds to quasinormal modes. The QNM spectrum consists of the continuous region where $N<4$. [The numerical values used for these plots, in units such that the healing length $\xi=1$, are $c=1 ; v=0.7$ in the subsonic region and $v=1.8$ in the supersonic region.] 

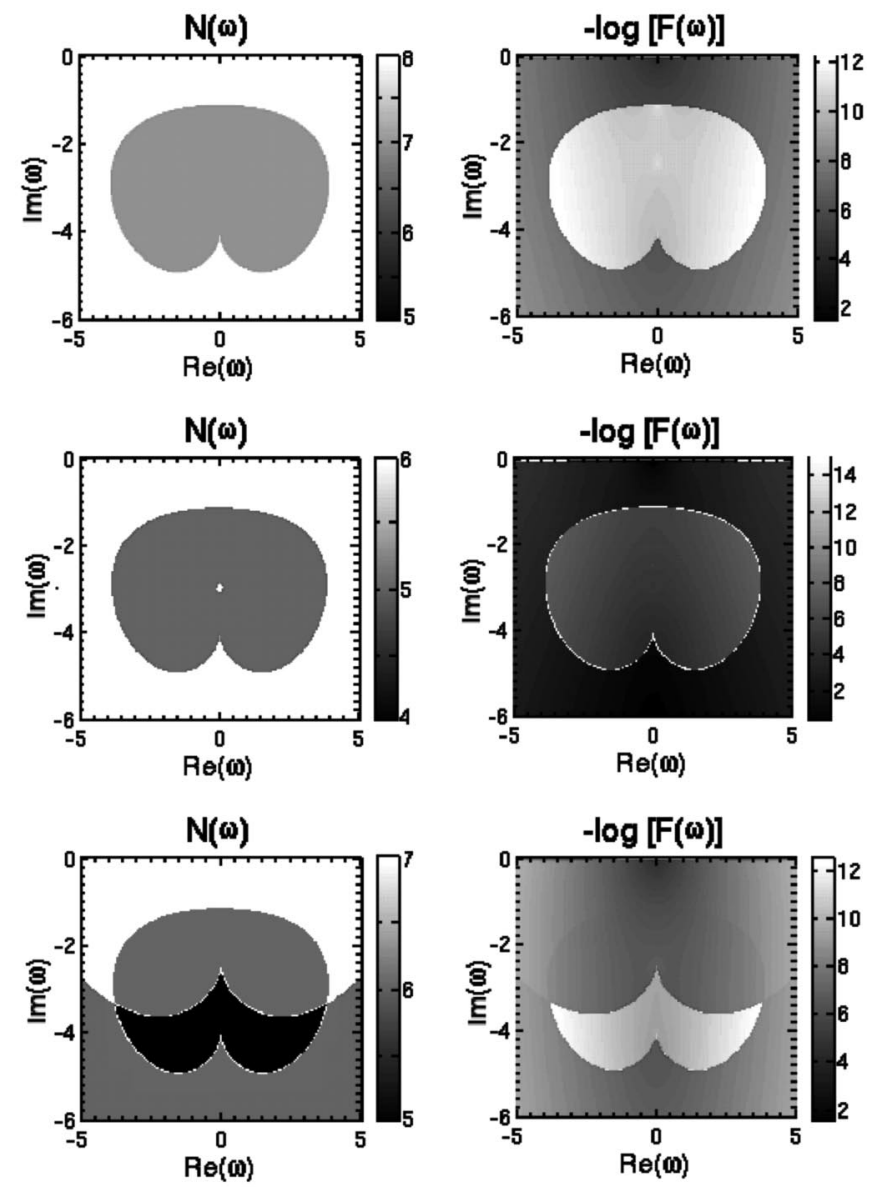

FIG. 3. Alteration of QNM spectrum resulting from imposing the additional boundary conditions of (from top to bottom) convergence in both asymptotic regions, in the right asymptotic region only, and in the left asymptotic region only. Since $F(\omega)$ never reaches zero, it is as if the QNM spectrum of Fig. 2 had disappeared. In other words, the QNMs are divergent in both asymptotic regions. [Same numerical values for $c$ and $v$ as in Fig. 2.]

them. This indicates that the continuous character of the QNM spectrum is an essential consequence of the modified dispersion relation, and not just of the particular characteristics of a precise configuration.

To sum up, there is a continuous region of frequencies $\omega$ such that they have associated modes

$$
u_{\omega}=e^{-i \omega t} \sum_{j=1}^{4} A_{j} e^{i k_{j} x}
$$

which are outgoing at both ends. These modes are divergent, just like in the hydrodynamic case, although the fact that here nonhydrodynamic modes come into play means that this relation cannot be derived directly from the dispersion relation.

\section{CONCLUSIONS}

We have examined the quasinormal modes of onedimensional black-hole configurations in BECs, and laid particular emphasis on the importance of the deviation of the full dispersion relation with respect to a relativistic or acoustic one. The full dispersion relation is quartic in BECs, as compared to the usual second order of its hydrodynamic and general relativistic equivalent. With such quadratic dispersion relations, outgoing relaxation modes are automatically divergent. In particular, this argument is valid for QNMs in GR black holes. But the modification of the dispersion relation means that this relation is no longer automatic. Nevertheless, the quasinormal modes in the systems discussed here are also divergent.

More importantly, the QNM spectrum that was obtained in these systems with a modified dispersion relation consists of a continuous region in the complex frequency plane. The importance of this result is easy to see when taking into account that both in GR and in BECs in the hydrodynamic limit, QNMs simply do not exist in $(1+1)$ dimensions, while in higher dimensions the quasinormal modes of a GR black-hole form a discrete spectrum. So due to the modification of the dispersion relation, the QNM spectrum in one-dimensional BEC black holes changes from nonexistent to a continuous region of frequencies.

We would like to end this paper with the straightforward speculation that the discrete spectrum which is obtained in the usual quasinormal mode analysis in standard $(3+1)$ $\mathrm{GR}$, will also develop continuous regions when taking the modification of the dispersion relation at high energies into account. The existence of these continuous bands could become a signal of trans-Planckian physics encoded in the emitted spectrum of gravitational waves.

\section{ACKNOWLEDGMENTS}

C. B. has been funded by the Spanish MEC under Project No. FIS2005-05736-C03-01 with a partial FEDER contribution. G. J. was supported by CSIC Grant No. I3P-BPD2005 of the I3P programme, cofinanced by the European Social Fund, and by the Spanish MEC under Project No. FIS2005-05736-C03-01. L. G. was supported by the Spanish MEC under Project No. FIS2005-05736C03-02. The authors also acknowledge support from the Spanish MEC Project No. FIS2006-26387-E. 
[1] C. Barceló, S. Liberati, and M. Visser, Living Rev. Relativity 8, 12 (2005), http://www.livingreviews.org/lrr2005-12.

[2] Artificial Black Holes, edited by M. Novello, M. Visser, and G. Volovik (World Scientific, Singapore, 2002).

[3] C. Barceló, S. Liberati, and M. Visser, Classical Quantum Gravity 18, 1137 (2001).

[4] L. J. Garay, J. R. Anglin, J. I. Cirac, and P. Zoller, Phys. Rev. Lett. 85, 4643 (2000).

[5] L. J. Garay, J. R. Anglin, J. I. Cirac, and P. Zoller, Phys. Rev. A 63, 023611 (2001).

[6] D. Mattingly, Living Rev. Relativity 8, 5 (2005), http:// www.livingreviews.org/lrr-2005-5.

[7] C. Barceló, A. Cano, L. J. Garay, and G. Jannes, Phys. Rev. D 74, 024008 (2006).

[8] K. D. Kokkotas and B. G. Schmidt, Living Rev. Relativity
2, 2 (1999), http://www.livingreviews.org/lrr-1999-2.

[9] H. P. Nollert, Classical Quantum Gravity 16, R159 (1999).

[10] E. Berti, V. Cardoso, and J. P. S. Lemos, Phys. Rev. D 70, 124006 (2004).

[11] Songbai Chen, Bin Wang, and Rukeng Su, Classical Quantum Gravity 23, 7581 (2006).

[12] F. Dalfovo, S. Giorgini, L. Pitaevskii, and S. Stringari, Rev. Mod. Phys. 71, 463 (1999).

[13] Y. Castin, in Coherent Atomic Matter Waves, Lecture Notes of Les Houches Summer School, edited by R. Kaiser, C. Westbrook, and F. David (EDP Sciences and Springer-Verlag, Berlin, 2001).

[14] N.D. Birrell and P.C.W. Davies, Quantum Fields In Curved Space (Cambridge University Press, Cambridge, 1982). 